\title{
Pathogenesis of Chronic Liver Injury and Hepatocellular Carcinoma in Alpha-1-Antitrypsin Deficiency
}

\author{
DAVID H. PERLMUTTER \\ Department of Pediatrics, University of Pittsburgh School of Medicine, Children's Hospital of Pittsburgh, Pittsburgh, PA 15213
}

\begin{abstract}
Alpha-1-antitrypsin (AT) deficiency is the most common genetic cause of liver disease in children. In addition to chronic liver inflammation and injury, it has a predilection to cause hepatocellular carcinoma later in life. The deficiency is caused by a mutant protein, ATZ, which is retained in the endoplasmic reticulum (ER) in a polymerized form rather than secreted into the blood in its monomeric form. The histologic hallmark of the disease is ATZ-containing globules in some, but not all, hepatocytes. Liver injury results from a gain-of-toxic function mechanism in which mutant ATZ retained in the ER initiates a series of pathologic events, but little is known about the mechanism by which this leads to carcinogenesis. Several recent observations from my laboratory have led to a novel hypothetical paradigm for carcinogenesis in AT deficiency in which globulecontaining hepatocytes are "sick," relatively growth suppressed, but also elaborating trans-acting regenerative signals. These signals are received and transduced by globule-devoid hepatocytes, which, because they are younger and have a lesser load of accumulated ATZ, have a selective proliferative advantage. Chronic regeneration in the presence of tissue injury leads to adenomas and ultimately carcinomas. Aspects of this hypothetical paradigm may also explain the proclivity for hepatocarcinogenesis in other chronic liver diseases, including other genetic diseases, viral hepatitis, and nonalcoholic steatohepatitis. (Pediatr Res 60: 233-238, 2006)
\end{abstract}

$\mathrm{A}^{\prime}$ lpha-1-antitrypsin (AT) is the principal blood-borne inhibitor of neutrophil elastase, a protease that can destroy the connective tissue matrix of the lung (1). It is considered a hepatic acute phase reactant because it is secreted by liver cells and its plasma levels increase during the host response to tissue injury/inflammation. The classic form of AT deficiency, which affects one in 1800 to 2000 livebirths, is associated with a mutant molecule termed ATZ that is retained in the ER of liver cells rather than secreted into the blood and body fluids. The mutation is thought to alter the usual folding pathway and to increase the likelihood that the protein polymerizes $(2,3)$.

Homozygotes are predisposed to premature development of pulmonary emphysema by a loss-of function mechanism in which the lack of AT in the lung permits uninhibited proteolytic damage to the connective tissue matrix $(4,5)$. Cigarette smoking markedly increases the risk and rate of development of emphysema (6), presumably because of functional inacti-

Received January 19, 2006; accepted March 21, 2006.

Correspondence: David H. Perlmutter, M.D., Department of Pediatrics, University of Pittsburgh School of Medicine, Children's Hospital of Pittsburgh, 3705 Fifth Avenue, Pittsburgh, PA 15213; e-mail: David.Perlmutter@chp.edu

Work from the author's laboratory described here was supported in part by NIH grants DK052565, HL037784, and DK061760. vation of residual AT by phagocyte-derived active oxygen intermediates. Other environmental factors and genetic traits also play a role in the variation in incidence and severity of lung disease among AT-deficient individuals (7).

Homozygotes are also at risk of liver disease. Indeed, AT deficiency is the most common genetic cause of liver disease in children (1) and also predisposes adults to chronic hepatitis and hepatocellular carcinoma (8). Further, the predilection for hepatocellular carcinoma among AT-deficient individuals is significantly greater than that attributable to cirrhosis alone (8). In contrast to the pathobiology of lung disease, liver injury in this deficiency involves a gain-of-toxic function mechanism whereby accumulation of the mutant ATZ molecule in the ER triggers a series of events that are eventually hepatotoxic. Evidence for the gain-of-toxic function mechanism comes from studies in which mice transgenic for the mutant human ATZ gene develop liver injury and hepatocellular carcinoma $(9,10)$. Because there are normal levels of antielastases in these mice, as directed by endogenous genes, the liver injury cannot be attributed to a loss-of-function mechanism.

Studies by Sveger $(11,12)$ in which every newborn in Sweden was screened for AT deficiency and then followed for $>30$ y have demonstrated the extraordinary variation in phenotypic expression of liver disease among homozygotes . These studies indicated that only $8-10 \%$ of this population developed clinically significantly liver disease over the first $20 \mathrm{y}$ of life. These data indicate that other genetic traits and/or environmental factors predispose a subgroup of homozygotes to liver injury or protect the remainder of the population from liver disease.

The histopathologic hallmark of the disease is intracellular globules in hepatocytes that stain with periodic acid-Schiff (PAS) after treatment with diastase. Early studies showed that these globules represent mutant ATZ in the ER of liver cells. Although these globules have been a focus of previous studies, there has been very limited investigation of the curious fact that many hepatocytes in these patients do not have globules. Studies of the Z\#2 mouse model, generated by using a human ATZ genomic fragment as transgene, have shown that these

Abbreviations: AT, alpha-1-antitrypsin; BrdU, bromedeoxyuridine; ER, endoplasmic reticulum; FAH mouse, fumarylacetoacetate hydrolase-deficient mouse; NTBC, 2-(2-nitro-4-trifluromethylbenzoyl)-1,3-cyclohexanedione; PAS, periodic acid-Schiff; UPR, unfolded protein response

DOI: $10.1203 / 01 . p d r .0000228350 .61496 .90$ 
globule-devoid cells occupy an increasing proportion of the liver as the animal ages and ultimately become the site of adenomas and carcinomas (13). In more recent studies using the PiZ mouse model, another transgenic mouse created with a human ATZ genomic fragment, we have shown that there is increased proliferation of these globule-devoid hepatocytes at a rate that is directly proportional to the number of globulecontaining hepatocytes (14). These observations have led to a novel paradigm in which the globule-containing hepatocytes engender a cancer-prone state by surviving with intrinsic damage and by chronically stimulating "in trans" adjacent relatively undamaged globule-devoid hepatocytes that have a selective proliferative advantage. The paradigm also appears to be relevant to the mechanism by which other chronic liver diseases are predisposed to hepatic cancer.

\section{MOLECULAR BASIS OF THE CLASSICAL FORM OF AT DEFICIENCY}

The ATZ molecule is characterized by a point mutation that results in the substitution of lysine for glutamate 342 , and this mutation accounts for its defective secretion. By adding a bulky side chain at the base of the reactive loop "scaffold," this substitution is thought to interfere with relaxation of the reactive loop into a gap in the A sheet of the AT molecule and, thereby, to increase the likelihood of polymerization by a mechanism in which reactive loops of adjacent ATZ molecules can insert into the gap in the A sheet, the so-called loop-sheet insertion mechanism $(15,16)$. This tendency to polymerize has been substantiated by observing polymers in the ER of liver cells by rotary electron microscopy of liver biopsy specimens from a patient with AT deficiency (16).

Several studies have suggested that polymerization of ATZ is the cause of its retention in the ER, including most notably studies in which secretion of ATZ is partially corrected by introduction of a second mutation that suppresses loop-sheet polymerization $(17,18)$. However, these studies cannot exclude the possibility that retention is caused by a distinct abnormality in folding that is also partially corrected by the second, experimentally introduced mutation. Indeed, several recent observations militate against the idea that polymerization is the cause of ER retention. First, naturally occurring variants of AT in which the carboxyl terminal tail of AT is truncated, including a double mutant with the substitution that characterizes the $\mathrm{Z}$ allele and the substitution that results in carboxyl terminal truncation, are retained in the ER even though they do not polymerize (19). Second, only $18 \%$ of the intracellular pool of ATZ at steady state is in the form of polymers in model cell lines characterized by marked ER retention $(19,20)$. This is because most of the cellular pool of ATZ in the ER in vivo is in heterogeneous, soluble complexes with multiple ER chaperones (19), a state that cannot be modeled by studies of purified ATZ in vitro. These observations suggest the possibility that polymerization of ATZ in the ER is an effect rather than a cause of the retention. As an effect, polymerization may still be very important in determining how ATZ is degraded, what cellular adaptive responses are activated, and how retention of ATZ leads to liver injury and carcinogenesis.

\section{CELLULAR ADAPTIVE RESPONSES TO MUTANT ATZ RETAINED IN THE ER}

We now know that cells have elaborate mechanisms for degrading mutant proteins that are retained in the ER. The pathway, or pathways, by which retained ATZ is degraded is also a candidate for genetic variations that predispose some homozygotes or protect other homozygotes from liver injury by the gain-of-toxic function mechanism. One study has provided a substantiation of this concept by showing that there is a lag in ER degradation of ATZ after gene transfer into cell lines derived from "susceptible hosts," homozygotes with liver disease, when compared with cell lines from "protected hosts," homozygotes completely free of liver disease (21).

Several different pathways appear to be involved in the ER degradation of ATZ. The proteosomal system was implicated first and has since been demonstrated in a number of different model systems (22-26). Both the classic ubiquitin-dependent and the ubiquitin-independent proteosomal pathways play a role (24). However, it is still not entirely clear how the ATZ on the luminal side of the ER membrane reaches the proteosome in the cytoplasm. Some luminal substrates have been shown to traverse the ER membrane to reach the cytoplasm by a retrograde translocation mechanism. However, the only evidence of this mechanism as a part of the ER degradation pathway for ATZ comes from the studies of Werner et al. (22) in which ATZ could be detected in the cytosolic fraction of yeast when the proteosome was inhibited, but it was only a minor fraction of the ATZ that was undergoing degradation. A mechanism in which the proteosome directly mediates extraction of substrates from the ER membrane, which has been described for model ER degradation substrates (27), represents one possible alternative.

There are at least several nonproteosomal pathways that contribute to ER degradation of ATZ as well (24). Cabral et $a l$. (28) have provided evidence for a nonproteosomal pathway that is sensitive to tyrosine phosphatase inhibitors. We have shown that macroautophagy contributes to the disposal of ATZ that is retained in the ER, using chemical inhibitors of autophagy (29) as well as cell lines that are genetically engineered for deficient autophagy (30). Macroautophagy is a general stress-activated degradative mechanism whereby cytosol and intracellular organelles are first enveloped by distinct, newly forming vesicles and then delivered to the lysosome for degradation. This process, which has been highly conserved in evolution, is activated by starvation and bacterial invasion and plays a role in differentiation, morphogenesis, and senescense. Recent studies in autophagy-deficient mammalian cell lines (30) and autophagy-deficient yeast (31) suggest that the autophagic response is particularly important for insoluble polymers/aggregates of ATZ when there are high levels of expression.

In addition to degradation mechanisms, cells appear to have a number of other mechanisms by which they attempt to adapt to, or protect themselves from, proteins that are retained in the 
ER. Because differences in an adaptive response could theoretically explain variation in the liver disease phenotype, we have recently begun a series of studies designed to characterize how cells respond to ER retention of ATZ using cell line and transgenic mouse model systems with tetracyclineinducible expression of the mutant gene. These models permit us to see the earliest responses and to separate them from compensatory adaptations that arise later. Because the relative expression of the mutant gene can be regulated in this kind of model system, it is also possible to determine the effect of expressing the mutant gene product at specific concentrations, at specific stages of development and for specific time intervals.

First, we found that accumulation of ATZ in the ER elicits mitochondrial dysfunction (32). It has long been known that mitochondria are closely apposed to the ER and recent studies have shown that there are elaborate mechanisms by which the ER and mitochondrion communicate with each other $(33,34)$. This mitochondrial injury was demonstrated functionally and by detailed ultrastructural studies in the liver of affected patients as well as in the model cell lines and transgenic mice. It is not yet clear whether it is directly mediated by the ER accumulation or indirectly results from an over-exuberant autophagic response. Antagonism of mitochondrial dysfunction by administration of cyclosporine $\mathrm{A}$ is associated with reduction in mitochondrial ultrastructural change, caspase-3 activation and liver disease morbidity in the PiZ transgenic mouse (32). These data raise the interesting possibility that the adaptive response actually contributes to liver injury by the release of active oxygen intermediates that accompanies mitochondrial dysfunction.

Second, we have found that accumulation of ATZ in the ER is sufficient to activate the autophagic response. This was demonstrated by mating two types of transgenic mice: the $\mathrm{Z}$ mouse with liver-specific inducible expression of ATZ in which ATZ accumulates in the ER of hepatocytes only when doxycycline is removed from the drinking water (35) and the GFP-LC3 mouse in which autophagosomes have green fluorescence because LC3 is an autophagosomal membranespecific protein (36). Although green fluorescent autophagosomes are only seen in the liver of the GFP-LC3 mouse when it is starved, they are seen in the liver of the $\mathrm{Z} \times$ GFP-LC3 mouse simply by removing doxycycline from the drinking water and inducing the accumulation of mutant ATZ in the ER of hepatocytes (29). This is a particularly important observation because it represents the first evidence that a single protein can activate the autophagic response.

To our great surprise, accumulation of ATZ in the ER does not activate the unfolded protein response (UPR). The UPR is a signaling pathway activating a number of genes in response to accumulation of unfolded proteins in the ER (37). In addition to new synthesis of ER chaperones, such as BiP, and enzymes that facilitate disulfide bond formation, bolstering the protein-folding capacity of the ER, and lipids for synthesis of new ER membrane required to handle the increased protein load, an increase in synthesis of proteins that participate in degradative and other cellular translocation mechanisms occurs. There is also a decrease in initiation of translation in such a way that only specific mRNAs can be translated and thereby preventing the de novo synthesis of proteins that will further accumulate in the ER (38). Although we do not detect activation of the UPR when ATZ accumulates in the ER, we do detect it when truncated nonpolymerogenic AT mutants accumulate in the ER, suggesting that it is the formation of polymers in the ER by ATZ that suppresses the UPR (35). We believe the lack of UPR signaling in response to the accumulation of ATZ in the ER is a very important part of the mechanism of carcinogenesis; activation of UPR signaling might be expected to lead to cell death, and lack of UPR signaling would permit survival of cells that have accumulated ATZ, the so-called sick but not dead, globule-containing hepatocytes (see below).

In contrast, the ER overload pathway and its signature target nuclear factor- $\kappa \mathrm{B}(\mathrm{NF}-\kappa \mathrm{B})$ are activated when ATZ accumulates in the ER (35). The ER overload pathway was first described as activation of the transcription factor NF- $\kappa \mathrm{B}$ in response to the type of "ER stress" that is generated by treatment of cells with brefeldin A or by experimental accumulation of adenovirus E3 protein in the ER (39). The fact that ER accumulation of ATZ activates NF- $\kappa$ B but not the UPR provides strong corroborating evidence of the previously held contention that the ER overload pathway was distinct from the UPR (39). Activation of NF- $\kappa$ B has potentially important implications for target organ injury in AT deficiency. First, through NF- $\kappa \mathrm{B}$, accumulation of ATZ in liver cells and respiratory epithelial cells (40) could mediate inflammation in the liver and the lung, particularly neutrophil infiltration in response to the NF- $\kappa \mathrm{B}$ target interleukin- 8 . Second, activation of NF- $\kappa \mathrm{B}$ has been shown to play a key role in inflammationassociated carcinogenesis (41-43) and therein could be involved in the pathogenesis of hepatocellular carcinoma in AT deficiency.

We also examined two other signal transduction pathways that have been associated with ER stress. For one, we found that ER accumulation of ATZ led to cleavage/activation of the ER caspases: caspase-12 in mouse cells and caspase- 4 in human cells (35). These results indicate that both the mitochondrial and ER caspase pathways are activated in AT deficiency. For another, we found that accumulation of ATZ in the ER specifically mediated cleavage/activation of BAP31 (35), an integral membrane protein of the ER that is involved in the ER retention of several proteins (44) and appears to mediate proapoptotic signals from the ER to mitochondria (45). This last result may provide a mechanistic basis for the mitochondrial dysfunction that was recently found in cell line and transgenic mouse models as well as in the liver of deficient patients (32).

\section{HEPATIC INJURY AND REGENERATION IN A MOUSE MODEL OF AT DEFICIENCY}

To begin to understand how the liver is injured and how it regenerates in AT deficiency, Rudnick et al. (14) used the PiZ mouse model of AT deficiency and measured the degree of injury by BrdU labeling to quantify hepatocellular proliferation. These studies showed that there was increased hepato- 
cellular proliferation in the liver of the PiZ mouse at baseline. Although the increase was five- to 10 -fold above the controls and highly statistically significant, there was still a relatively low number of BrdU-positive hepatocytes at one time (2\% to $3 \%$ detected over $72 \mathrm{~h}$ of continuous labeling). These data indicate that liver injury in the mouse model is relatively mild and appropriately corresponds to the smoldering and slowly progressing liver disease that is seen in most AT-deficient patients. The increase in hepatocellular proliferation was entirely accounted for by male mice and correlated with increased number of hepatocytes that had PAS-positive, diastase-resistant globules as well as increased steady state levels of AT mRNA and protein. Systemic administration of testosterone to female PiZ mice led to an increase in number of globule-containing hepatocytes, steady-state levels of AT mRNA and protein, and an increase in BrdU labeling that was comparable to that in male PiZ mice. These results were consistent with those of previous studies showing that androgens had a positive regulatory effect on the human AT gene; in this mouse, that would represent a positive regulatory effect on the human transgene. However, more importantly, these results indicated that the increase in hepatocellular proliferation in the PiZ mouse liver was proportional to the number of globule-containing cells and/or the level of ATZ accumulation within these cells.

Next, double labeling for PAS-staining and BrdU-staining was used to determine whether the globule-containing or the globule-devoid cells were proliferating. The results showed that almost all the BrdU-positive cells in the PiZ mouse liver were globule devoid and, conversely, very few of the globulecontaining cells were BrdU positive. These results provided evidence that the globule-devoid hepatocytes have a selective proliferative advantage in the PiZ liver.

To further characterize this selective proliferative advantage, the hepatocellular proliferative response to partial hepatectomy in the PiZ mice was determined. Although partial hepatectomy resulted in increased mortality among PiZ mice as compared with their C57/BL6 nontransgenic littermates, those PiZ mice that survived had a similar proliferative response to the C57 littermates. In particular, there was a comparable increase in BrdU labeling of globule-containing and globule-devoid hepatocytes. These data indicated that the block in proliferation of globule-containing cells is relative; that is, when the stimulus was as powerful as the one that follows partial hepatectomy, those cells were able to replicate.

Finally, these studies showed that there was increased caspase- 9 and caspase- 3 activation in the PiZ mouse liver. Together with the observation that the liver-to-body-weight ratio in PiZ mice was identical to that observed in wild-type mice, these data suggested that hepatocytes undergo programmed cell death at a rate equivalent to the rate of increased cellular proliferation. However, increased apoptosis was not detected either histologically or by TUNEL staining in the livers of these animals. Thus, the increase in cell death that must be occurring in the PiZ mouse liver is at a rate that is lower than the limits for detection of apoptosis using currently available in situ methods. This is, again, consistent with the slowly progressing nature of the disease. Most informative, however, is the lack of apoptosis histologically and by TUNEL staining of cells that obviously have globules. If we take this together with our data on how cells respond to accumulation of ATZ, we would suggest that the globulecontaining cells are "sick but not dead"- - they have activated ER- and mitochondrial-caspases, NF- $\kappa \mathrm{B}$, and autophagy with a relative block in proliferation, but they are not apoptotic.

\section{A NOVEL HYPOTHETICAL PARADIGM FOR HEPATOCELLULAR CARCINOMA IN AT DEFICIENCY}

These data have led to a hypothetical paradigm in which the accumulation of ATZ in the ER activates ER and mitochondrial caspases, NF- $\kappa \mathrm{B}$, and autophagy, but the caspase pathway is blocked at terminal steps so the globule-containing hepatocytes are "sick but not dead." An "injury/regeneration" signal is generated by these cells in proportion to the amount of ATZ accumulation per cell or the number of cells with ATZ accumulation. Hepatocytes with lesser amounts of ATZ, globule-devoid hepatocytes are therein chronically stimulated "in trans" to divide. The cancer-prone state is then engendered by having cells that are unable to die at the appropriate time and cells that are chronically dividing in an inflamed milieu. The paradigm anticipates that some of the globule-containing hepatocytes eventually die, but, because the block in their proliferation is relative, they can be replenished, at least to the extent that there are always some of these cells present in the affected liver. The mechanism by which globule-devoid hepatocytes arise in unknown, but one possibility is that they are progenitors or relatively young cells for which there has not been enough time to accumulate as much ATZ. Furthermore, because of the proliferative advantage, it would presumably only take one or a few of these to ultimately lead to many more.

This model is consistent with the observations of Geller $e t$ al. (13), using the Z\#2 transgenic mouse model of AT deficiency. These authors found that increasing areas of the liver were negative for AT immunostaining as the mice aged so that by age $12 \mathrm{mo},>90 \%$ of the liver was AT negative, corresponding to the globule-devoid hepatocytes. Moreover, by 6 mo, adenomas began developing in the AT-negative areas and by $18 \mathrm{mo},>80 \%$ of these mice had hepatocellular carcinoma arising within the AT-negative areas $(13,46)$.

Why adenomas and carcinomas arise rather specifically from the AT-negative regions is not clear. There are many possible explanations. Certainly, this is where the most rapid cell proliferation is occurring. The known antitumorigenic effect of autophagy, which is relatively specifically activated in the globule-containing hepatocytes of the PiZ mice and PIZZ patients (29), also may play a role $(47,48)$.

The mechanism by which the caspase pathway is blocked in globule-containing hepatocytes is not known, but one possibility is the antiapoptotic effect of heat shock proteins (49). We have shown that accumulation of ATZ leads to an increase in expression of several heat shock proteins (50).

A similar model may be involved in the mechanism of hepatocarcinogenesis in the fumarylacetoacetate hydrolase- 
deficient (FAH) mouse model of tyrosinemia (51). In these studies, the liver of the FAH mouse was found to contain hepatocytes that were damaged but not dead. In fact, these cells were found to be resistant to cell death and, therefore, prone to carcinogenesis. By the paradigm proposed here, these hepatocytes would be considered equivalent to the globulecontaining hepatocytes in the PiZ mouse liver. They are damaged by an entirely different mechanism than the globulecontaining hepatocytes, and the damage is much more severe and rapidly progressing. However, they share the property of being TUNEL-negative, and up-regulation of antiapoptotic heat shock proteins has also been implicated in the block in their cell death (51). There is also evidence in the FAH mouse of chronic stimulation in "trans" of hepatocytes that are not damaged and have a selective proliferative advantage. According to the paradigm we are proposing here, these hepatocytes correspond to the globule-devoid hepatocytes in the PiZ mouse liver. The mechanism by which some undamaged hepatocytes arise in the FAH mouse as well as in tyrosinemic patients is known and appears to involve mutation reversion (52), whereas the origin/mechanism of globule-devoid hepatocytes in the PiZ mouse and AT-deficient patient is still unknown. Evidence of a "trans" effect in the FAH mouse comes from studies with transplanted normal hepatocytes that are capable of undergoing multiple rounds of replication in the FAH mouse liver but only if disease is present. Once the mouse is given the drug NTBC, which prevents the accumulation of toxic intermediates in the damaged cells, transplanted hepatocytes will no longer replicate in the FAH mouse liver (53). However, at least one part of this paradigm does not fit with what has been found in humans with hereditary tyrosinemia, which is that dysplasia and carcinoma have only been found to arise from the damaged cell compartment (54). It is possible that this difference relates to the net balance of carcinogenic and anticarcinogenic factors in each compartment in the two different diseases. For instance, activation of $\mathrm{NF}-\kappa \mathrm{B}$ and of autophagy is present in the damaged cell compartment in the AT deficiency models but not in the tyrosinemia model.

A similar situation has been described for hepatocarcinogenesis in hepatitis B surface antigen (HBsAg) transgenic mice (55). In this model of hepatitis B-associated liver injury, like the PiZ model, the degree of hepatocellular proliferation correlated with the presence of injury and carcinomas and with the cellular load of HBsAg, but the proliferating cells, adenomas, and carcinomas had significantly lesser amounts of retained HBsAg. However, because this transgenic model only expresses HBsAg and not complete hepatitis B virus, it is not clear whether the model truly reflects hepatocarcinogenesis in chronic hepatitis B virus infection. It is of some interest that HBsAg is known to be selectively retained in the ER of hepatocytes, and this process is thought to be responsible for the so-called ground glass hepatocyte (56). Prolonged ER retention has also been observed for several proteins encoded by hepatitis $C$ virus in infected hepatocytes (57), raising the possibility that a similar paradigm applies to cancer predisposition in hepatitis $C$ infection. Several studies have shown an increase in the proliferation of hepatocytes with lesser loads of fat accumulation in mouse models of obesity-related hepatic steatosis (58). Moreover, these cells have some of the staining characteristics of a type of progenitor cell known as the oval cell (59). Thus, the paradigm in which hepatocarcinogenesis involves cross-talk between inappropriately surviving, damaged cells and younger cells with a selective proliferative advantage may be applicable to chronic liver disease due to viral infections or associated with obesity in addition to genetic liver disease.

Acknowledgment. The author is indebted to Dr. Harvey R. Colten for inspiration and masterful mentoring for more than 20 years.

\section{REFERENCES}

1. Huber R, Carrell RW 1989 Implications of the three-dimensional structure of alpha 1-antitrypsin for structure and function of serpins. Biochemistry 28:8951-8966

2. Perlmutter DH 2002 Liver injury in $\alpha 1$-antitrypsin deficiency: an aggregated protein induces mitochondrial injury. J Clin Invest 110:1579-1583

3. Perlmutter DH $2001 \alpha 1$-Antitrypsin deficiency. In: Arias IM, Chisari FV, Shafritz DA, Boyer JL, Fausto N (eds) Liver, Biology and Pathobiology. Raven Press, New York, pp 699-719

4. Janoff A 1985 Elastases and emphysema: current assessment of the proteaseantiprotease hypothesis. Am Rev Respir Dis 132:417-433

5. Crystal RG 1990 Alpha 1-antitrypsin deficiency, emphysema and liver disease: genetic basis and strategies for therapy. J Clin Invest 95:1343-1352

6. Janus ED, Phillips NT, Carrell RW 1985 Smoking, lung function and $\alpha 1$-antitrypsin deficiency. Lancet 1:152-154

7. Silverman EK, Province MA, Rao DC, Pierce JA, Campbell EJ 1990 A family study of the variability of pulmonary function in $\alpha 1$-antitrypsin deficiency: quantitative phenotypes. Am Rev Respir Dis 142:1015-1021

8. Eriksson S, Carlson J, Velez R 1986 Risk of cirrhosis and primary liver cancer in $\alpha 1$-antitrypsin deficiency. N Engl J Med 314:736-739

9. Carlson JA, Rogers BB, Sifers RN, Finegold MJ, Clift SM, DeMayo FJ, Bullock DW, Woo SL 1989 Accumulation of PiZ antitrypsin causes liver damage in transgenic mice. J Clin Invest 83:1183-1190

10. Dycaico MJ, Grant SC, Felts K, Nichols WS, Geller SA, Hager JH, Pollard AJ, Kohler SW, Short HP, Jirik FR, Hanahan D, Sorge JA 1988 Neonatal hepatitis induced by $\alpha 1$-antitrypsin: a transgenic mouse model. Science 242:1409-1412

11. Sveger T 1976 Liver disease in $\alpha 1$-antitrypsin deficiency detected by screening of 200,000 infants. N Engl J Med 294:1316-1321

12. Sveger T 1998 The natural history of liver disease in $\alpha 1$-antitrypsin deficient children. Acta Paediatr Scand 77:847-851

13. Geller SA, Nichols WS, Kim S, Tolmachoff T, Lee S, Dycaico MJ, Felts K, Sorge JA 1994 Hepatocarcinogenesis is the sequel to hepatitis in Z\#2 $\alpha 1$-antitrypsin transgenic mice: histopathological and DNA ploidy studies. Hepatology 19:389-397

14. Rudnick DA, Liao Y, An JK, Muglia LJ, Perlmutter DH, Teckman JH 2004 Analyses of hepatocellular proliferation in a mouse model of $\alpha 1$-antitrypsin deficiency. Hepatology 39:1048-1055

15. Lomas DA, Mahadeva R $2002 \alpha 1$-antitrypsin polymerization and the serpinopathies: pathobiology and prospects for therapy. J Clin Invest 110:1585-1590

16. Lomas DA, Evans DL, Finch JT, Carrell RW 1992 The mechanism of Z $\alpha 1$ antitrypsin accumulation in the liver. Nature 357:605-607

17. Kim J, Lee KN, Yi GS, Yu MH 1995 A thermostable mutation located at the hydrophobic core of $\alpha 1$-antitrypsin suppresses the folding defect of the Z-type variant. J Biol Chem 270:8597-8601

18. Sidhar SK, Lomas DA, Carrell RW, Foreman RC 1995 Mutations which impede loop-sheet polymerization enhance the secretion of human alpha 1-antitrypsin deficiency variants. J Biol Chem 270:8393-8396

19. Lin L, Schmidt B, Teckman J, Perlmutter DH 2001 A naturally occurring nonpolymerogenic mutant of $\alpha 1$-antitrypsin characterized by prolonged retention in the endoplasmic reticulum. J Biol Chem 276:33893-33898

20. Schmidt BZ, Perlmutter DH 2005 GRP78, GRP94 and GRP170 interact with $\alpha 1$ AT mutants that are retained in the endoplasmic reticulum. Am J Physiol Gastrointest Liver Physiol 289:G444-G455

21. Wu Y, Whitman I, Molmenti E, Moore K, Hippenmeyer P, Perlmutter D 1994 A lag in intracellular degradation of mutant $\alpha 1$-antitrypsin correlates with the liver disease phenotype in homozygous PiZZ $\alpha 1$-antitrypsin deficiency. Proc Natl Acad Sci U S A 91:9014-9018

22. Werner ED, Brodsky JL, McCracken AA 1996 Proteasome-dependent endoplasmic reticulum-associated protein degradation: an unconventional route to a familiar fate. Proc Natl Acad Sci U S A 93:13797-13801

23. Qu D, Teckman JH, Omura S, Perlmutter DH 1996 Degradation of mutant secretory protein, $\alpha 1$-antitrypsin $\mathrm{Z}$, in the endoplasmic reticulum requires proteasome activity. J Biol Chem 271:22791-22795

24. Teckman JH, Gilmore R, Perlmutter DH 2000 Role of ubiquitin in proteasomal degradation of mutant $\alpha 1$-antitrypsin $\mathrm{Z}$ in the endoplasmic reticulum. Am J Physiol Gastrointest Liver Physiol 278:G39-G48 
25. Teckman JH, Burrows J, Hidvegi T, Schmidt B, Hale PD, Perlmutter DH 2001 The proteasome participants in degradation of mutant $\alpha 1$-antitrypsin $\mathrm{Z}$ in the endoplasmic reticulum of hepatoma-derived hepatocytes. J Biol Chem 276:44865-44872

26. Cabral CM, Liu Y, Moremen KW, Sifers RN 2002 Organizational diversity among distinct glycoprotein endoplasmic reticulum-associated degradation programs. Mol Biol Cell 13:2639-2650

27. Mayer TU, Braun T, Jentsch S 1998 Role of the proteasome in membrane extraction of a short-lived ER-transmembrane protein. EMBO J 17:3251-3257

28. Cabral CM, Choudhury P, Liu Y, Sifers RN 2000 Processing by endoplasmic reticulum mannosidases partitions a secretion-impaired glycoprotein into distinct disposal pathways. J Biol Chem 275:25015-25022

29. Teckman JH, Perlmutter DH 2000 Retention of mutant alpha (1)-antitrypsin Z in endoplasmic reticulum is associated with an autophagic response. Am J Physiol Gastrointest Liver Physiol 279:G961-G974

30. Kamimoto T, Shoji S, Mizushima N, Umegayashi K, Hidvegi T, Perlmutter DH, Yoshimori T 2006 Intracellular inclusions containing mutant $\alpha 1$-antitrypsin $\mathrm{Z}$ are propagated in the absence of autophagic activity. J Biol Chem 281:4467-4476

31. Kruse KB, Brodsky JL, McCracken AA 2006 Characterization of an ERAD gene as VPS30/ATG6 reveals two alternative and functionally distinct protein quality control pathways: one for soluble A1PiZ and another for aggregates of A1PiZ. Mol Biol Cell 17:203-212

32. Teckman JH, An JK, Blomenkamp K, Schmidt B, Perlmutter D 2004 Mitochondrial autophagy and injury in the liver in $\alpha 1$-antitrypsin deficiency. Am J Physiol Gastrointest Liver Physiol 286:G851-G862

33. Perkins G, Renken C, Martone ME, Young SJ, Ellisman M, Frey T 1997 Electron tomography of neuronal mitochondria: three-dimensional structure and organization of cristae and membrane contacts. J Struct Biol 119:260-272

34. Achleitner G, Gaigg B, Krasser A, Kainersdorfer E, Kohlwein SD, Perktold A, Zellnig G, Daum G 1999 Association between the endoplasmic reticulum and mitochondria of yeast facilitates intraorganelle transport of phospholipids through membrane contact. Eur J Biochem 264:545-553

35. Hidvegi T, Schmidt BZ, Hale P, Perlmutter DH 2005 Accumulation of mutant alpha-1-antitrypsin $\mathrm{Z}$ in the ER activates caspases- 4 and $-12, \mathrm{NF} \kappa \mathrm{B}$ and BAP31 but not the unfolded protein response. J Biol Chem 280:39002-39015

36. Mizushima N, Yamamoto A, Matsui M, Yoshimori T, Ohsumi Y 2004 In vivo analysis of autophagy in response to nutrient starvation using transgenic mice expressing a fluorescent autophagosome marker. Mol Biol Cell 15:1101-1111

37. Zhang K, Kaufman RJ 2004 Signaling the unfolded protein response from the endoplasmic reticulum. J Biol Chem 279:25935-25938

38. Ron D 2002 Translational control in the endoplasmic reticulum stress response. J Clin Invest 110:1383-1388

39. Pahl HL, Sester M, Burgert HG, Baeuerle PA 1996 Activation of transcription factor $\mathrm{NF} \kappa \mathrm{B}$ by the adenovirus $\mathrm{E} 3 / 19 \mathrm{~K}$ protein requires its ER retention. J Cell Biol 132:511-522

40. Hu C, Perlmutter DH 2002 Cell-specific involvement of HNF- $1 \beta$ in $\alpha 1$-antitrypsin gene expression in human respiratory epithelial cells. Am J Physiol Lung Cell Mol Physiol 282:L757-L765

41. Pikarsky E, Porat RM, Stein I, Abramovitch R, Amit S, Kasem S, Gutkovich-Pyest E, Urieli-Shoval S, Galun E, Ben-Neriah Y $2004 \mathrm{NF} \kappa \mathrm{B}$ functions as a tumor promoter in inflammation-associated cancer. Nature 431:461-466

42. Greten FR, Eckman L, Greten TF, Park JM, Li ZW, Egan LJ, Kagnoff MF, Karin M 2004 IKK $\beta$ links inflammation and tumorigenesis in a mouse model of colitisassociated cancer. Cell 118:285-296
43. Maeda S, Kamata H, Luo J-L, Leffert H, Karin M 2005 IKK $\beta$ couples hepatocyte death to cytokine-driven compensatory proliferation that promotes chemical hepatocarcinogenesis. Cell 121:977-990

44. Schamel WW, Kuppig S, Becker B, Gimborn K, Hauri HP, Reth MA 2003 A high-molecular-weight complex of membrane proteins BAP29/BAP31 is involved in the retention of membrane-bound $\mathrm{IgD}$ in the endoplasmic reticulum. Proc Natl Acad Sci U S A 100:9861-9866

45. Breckenridge DG, Stojanovic M, Marcellus RC, Shore GC 2003 Caspase cleavage product of BAP31 induces mitochondrial fission through endoplasmic reticulum calcium signals, enhancing cytochrome $\mathrm{c}$ release to the cytosol. J Cell Biol 160:1115-1127

46. Geller SA, Nichols WS, Dycaico MJ, Felts KA, Sorge JA 1990 Histopathology of $\alpha 1$-antitrypsin liver disease in a transgenic mouse model. Hepatology 12:40-47

47. Qu X, Yu J, Bhagat G, Furuya N, Hibshoosh H, Troxel A, Rosen J, Eskelinen E-L, Mizushima N, Ohsumi Y, Cattoretti G, Levine B 2003 Promotion of tumorigenesis by heterozygous disruption of the beclin1 autophagy gene. J Clin Invest 112:18091820

48. Yue Z, Jin S, Yang C, Levine AJ, Heintz N 2003 Beclin 1, an autophagy gene essential for early embryonic development, is a haploinsufficient rumor suppressor. Proc Natl Acad Sci U S A 100:15077-15082

49. Bruey JM, Ducasse C, Bonniaud P, Ravagnan L, Susin SA, Diaz-Latoud C, Gurbuxani S, Arrigo AP, Kroemer G, Solary E, Garrido C 2000 Hsp27 negatively regulates cell death by interacting with cytochrome c. Nat Cell Biol 2:645-652

50. Perlmutter DH, Schlesinger MJ, Pierce JA, Punsal PI, Schwartz AL 1989 Synthesis of stress proteins is increased in individuals with homozygous PiZZ $\alpha 1$-antitrypsin deficiency and liver disease. J Clin Invest 84:1555-1561

51. Vogel A, van Den Berg IE, Al-Dhalimy M, Groopman J, Ou CN, Ryabinina O, Iordanov MS, Finegold M, Grompe M 2004 Chronic liver disease in murine hereditary tyrosinemia type 1 induces resistance to cell death. Hepatology 39:433443

52. Kvittingen EA, Rootwelt H, Berger R, Brandtzaeg P 1994 Self-induced correction of the genetic defect in tyrosinemia type I. J Clin Invest 94:1657-1661

53. Overturf K, Al-Dhalimy M, Tanguay R, Brantly M, Ou CM, Finegold M, Grompe M 1996 Hepatocytes corrected by gene therapy are selected in vivo in a murine model of hereditary tyrosinaemia type I. Nat Genet 12:266-273

54. Demers SI, Russo P, Lettre F, Tanguay RM 2003 Frequent mutation reversion inversely correlates with clinical severity in a genetic liver disease, hereditary tyrosinemia. Hum Pathol 34:1313-1320

55. McLachlan A, Milich DR, Raney AK, Riggs MG, Hughes JL, Sorge J, Chisari FV 1987 Expression of hepatitis B virus surface and core antigens: influences of pre-S and precore sequences. J Virol 61:683-692

56. Wang HC, Wu HC, Chen CF, Fausto N, Lei HY, Su IJ 2003 Different types of ground glass hepatocytes in chronic hepatitis B virus infection contain specific pre-S mutants that may induce endoplasmic reticulum stress. Am J Pathol 163:2441-2449

57. Dubuisson J 2000 Folding, assembly and subcellular localization of hepatitis $\mathrm{C}$ virus glycoproteins. Curr Top Microbiol Immunol 242:135-148

58. Yang S, Lin HZ, Hwang J, Chacko VP, Diehl AM 2001 Hepatic hyperplasia in noncirrhotic fatty livers: is obesity-related hepatic steatosis a premalignant condition? Cancer Res 61:5016-5023

59. Roskams T, Yang SQ, Koteish A, Durnez A, DeVos R, Huang X, Achten R Verslype C, Diehl AM 2003 Oxidative stress and oval cell accumulation in mice and humans with alcoholic and nonalcoholic fatty liver disease. Am J Pathol 163:13011311 